\title{
Charge transfer in the weak driving force limit in blends of MDMO-PPV and dithienylthiazolo $[5,4-d]$ thiazoles towards organic photovoltaics with high $V_{\text {OC }}^{\dagger}$
}

\author{
Nissy Nevil, ${ }^{* a}$ Yun Ling, ${ }^{a}$ Sarah Van Mierloo, ${ }^{b}$ Jurgen Kesters, ${ }^{b}$ \\ Fortunato Piersimoni, ${ }^{c}$ Peter Adriaensens, ${ }^{b}$ Laurence Lutsen, ${ }^{d}$ Dirk Vanderzande, ${ }^{b d}$ \\ Jean Manca, ${ }^{c}$ Wouter Maes, ${ }^{b}$ Sabine Van Doorslaer ${ }^{a}$ and Etienne Goovaerts ${ }^{* a}$
}

Received 14th July 2012, Accepted 4th October 2012

DOI: $10.1039 / \mathrm{c} 2 \mathrm{cp} 42399 \mathrm{e}$

A series of three 5'-aryl-2,5-dithienylthiazolo[5,4- $d$ ] thiazole (DTTzTz) semiconducting molecules with different aryl substituents has been investigated as alternative acceptor materials in combination with the donor polymer poly[2-methoxy-5-(3',7'-dimethyloctyloxy)-1,4-phenylene vinylene] (MDMO-PPV) in order to evaluate the photoinduced charge transfer (CT) efficiency in the resulting blends, designed towards possible application in organic photovoltaics. Photoluminescence quenching together with polaron detection by electron paramagnetic resonance and photoinduced absorption (PIA) demonstrate an increasing charge transfer efficiency when the DTTzTz substituents are varied from thien-2-yl to 4-trifluoromethylphenyl and 4-cyanophenyl groups, correlating well with the increasing acceptor strength in this series of molecules. In line with this observation, there is a decrease in the effective optical bandgap relative to pure MDMO-PPV that becomes more pronounced along this series of acceptor compounds, reaching $0.12 \mathrm{eV}$ in the blend with 4-CN-Ph-DTTzTz. Intermolecular interactions between the blend components lead to lower energy transitions which are found to contribute significantly to the device external quantum efficiency. The high $V_{\mathrm{OC}}$ reached in devices based on MDMO-PPV:4-CN-Ph-DTTzTz blends meets the expectations for such a donor:acceptor combination. However, thermal activation of charge carrier recombination occurs because of the weak driving force for charge transfer, as shown by time-dependent PIA measurements, and this is suggested as a cause for the observed low photovoltaic performance.

\section{Introduction}

Organic semiconductors continue to attract attention as new functional materials for various electronic applications due to

\footnotetext{
${ }^{a}$ University of Antwerp, Physics Department, Universiteitsplein 1, B-2610 Antwerpen, Belgium.E-mail: Etienne Goovaerts@ua.ac.be

${ }^{b}$ Hasselt University, Institute for Materials Research

(IMO-IMOMEC), Design \& Synthesis of Organic Semiconductors (DSOS), Agoralaan 1 - Building D,

B-3590 Diepenbeek,Belgium.E-mail:Wouter.Maes@uhasselt.be ${ }^{c}$ Hasselt University, Institute for Materials Research

(IMO-IMOMEC), Organic + Nanostructured Electronics and Energy

Conversion $\left(\mathrm{ONE}^{2}\right)$, Universitaire Campus - Wetenschapspark 1, B-3590

Diepenbeek, Belgium.E-mail: Jean.Manca@uhasselt.be

${ }^{d}$ IMEC, IMOMEC Ass. Lab., Universitaire Campus - Wetenschapspark

1, B-3590 Diepenbeek, Belgium

$\dagger$ Electronic supplementary information (ESI) available: Table S1: profilometer thickness measurements of spin-cast films; figures: optical absorption and PL spectra of the pure compounds in solution (S1) and in spin-cast films (S2); X-band LI-EPR spectra of $1: 1$ and $4: 1$ polymer : DTTzTz films (S3); time-dependent PIA in $1: 1$ blends of MDMO-PPV with 4-CN-Ph-DTTzTz and with PCBM and their temperature dependence (S4). See DOI: 10.1039/c2cp42399e
}

their capability of varying the electronic function by smooth structural modifications and intermolecular interactions. ${ }^{1}$ Although there are many p-type semiconductors around small molecules ${ }^{2,3}$ as well as a steadily growing number of conjugated polymers ${ }^{4}$ - the class of acceptor materials applied in organic (opto)electronic applications continues to be dominated by fullerenes (in particular PCBM or [6,6]-phenyl- $\mathrm{C}_{61}$ butyric acid methyl ester and its derivatives). While fullerenes have undoubtedly been very successful as acceptors in photovoltaic applications, ${ }^{5,6}$ certain factors such as poor (red light) absorption, high cost and limited versatility of fullerene chemistry in comparison to conjugated organic compounds have motivated the search for alternative acceptor materials. In this respect, there are a number of examples in the literature that illustrate the use of non-fullerene small molecule electron acceptors in organic photovoltaics (OPV). ${ }^{3,7}$ Potential advantages of such conjugated molecular acceptors, besides the more generic and versatile synthesis, would be an increased optical absorption in the active layer and more opportunities for engineering of the bandgap in the acceptor material (e.g. for the collection of the higher-energy 
photons in tandem cells with optimal open-circuit voltage) and for stabilization strategies of the blend morphology.

There are numerous reports on prominent organic (small molecule) semiconductors for OPV and organic field-effect transistor (OFET) applications which are obtained by the modification of thiophene materials, and their properties have been improved by introducing additional substituents to the conjugated $\pi$ systems. Among the various acceptor molecules reported so far, the thiazolo[5,4- $d]$ thiazole (TzTz) unit is particularly attractive since it exhibits good electron acceptor properties, high oxidative stability and a strong tendency for $\pi-\pi$ stacking. ${ }^{8}$ In general, TzTz derivatives without additional alkyl substituents are poorly soluble and require vacuum deposition techniques to fabricate devices. The introduction of alkyl chains leads to a significantly improved solubility, enabling solution processing. Moreover, functionalization, purification and characterization also become considerably more straightforward. A series of highly soluble functionalized 2,5-dithienylthiazolo[5,4- $d$ ] thiazoles (DTTzTz) was recently synthesized within one of our groups and these expanded semiconductors were investigated as active materials for solution-processable OFETs. ${ }^{9-11}$ In the OPV field, a number of low bandgap copolymer structures incorporating $\mathrm{TzTz}$ moieties as the electron poor units have very recently appeared in the literature, showing rather high charge-carrier mobilities and power conversion efficiencies. ${ }^{12,13}$

At present the most successful organic solar cells are based on the 3-dimensional bulk heterojunction (3D BHJ) approach in which the active layer consists of two interpenetrating networks of an electron donor compound, most often a conjugated polymer, and an electron acceptor, in many cases fullerene derivatives. Photon absorption produces excitons, which at the donor-acceptor (D-A) interface are efficiently split by a charge transfer (CT) process with high quantum efficiency, approaching unity in specific polymer-fullerene blends. This is a first and important step in the photovoltaic process.
Spectroscopic techniques, and in particular photoluminescence (PL) quenching, ${ }^{14-17}$ light-induced electron paramagnetic resonance (LI-EPR) $^{14,16,18-21}$ and photoinduced absorption (PIA) ${ }^{14,18,22,23}$ have been used in a number of studies to characterise this process. Indeed, it was found that the forward electron transfer process occurs in the sub-picosecond time domain, ${ }^{24}$ faster than any competing process such as radiative recombination. The fact that the CT process quenches the PL of the conjugated polymer has been harnessed as a tool to characterise CT. ${ }^{15,25}$ In some cases, PL quenching might also occur due to a nonradiative mechanism or by energy transfer, ${ }^{26}$ which cannot be easily distinguished from the CT process. In such cases, LI-EPR and PIA are very sensitive and effective techniques measuring the resulting radicals in both the donor and acceptor compounds, allowing to differentiate CT from other processes.

As observed in BHJ solar cell devices with various D-A combinations, the open circuit voltage $\left(V_{\mathrm{OC}}\right)$ is found to be strongly correlated with the energy difference between the LUMO of the acceptor and the HOMO in the donor. ${ }^{27,28}$ Maximizing $V_{\text {OC }}$ by increasing this energy difference however tends to decrease the driving force for charge separation at the D-A interface. Indeed, a type II alignment of the bands is required with a sufficient energy gain to overcome the exciton binding energy and to favour charge separation. Several investigations have been reported recently on solar cell operation under low driving force conditions, exploring this as a route to higher efficiency in $\mathrm{BHJ}$ devices. ${ }^{29,30}$

In this work, we have selected three soluble functionalized dithienylthiazolo[5,4- $d]$ thiazole molecules, Th-DTTzTz, 4CF3-Ph-DTTzTz and 4-CN-Ph-DTTzTz (Fig. 1) as possible small molecule acceptors that possess decreasing LUMO energies throughout this series, as previously shown experimentally by cyclic voltammetry (CV) and supported by density functional theory (DFT) calculations. ${ }^{9,10}$ We report here the optical and electronic characterization of the blends of these DTTzTz compounds with the reference donor polymer<smiles>[Z17]c1cc(-c2cccs2)sc1-c1nc2sc(-c3sc(-c4cccs4)cc3CCCCCCCC)nc2s1</smiles>

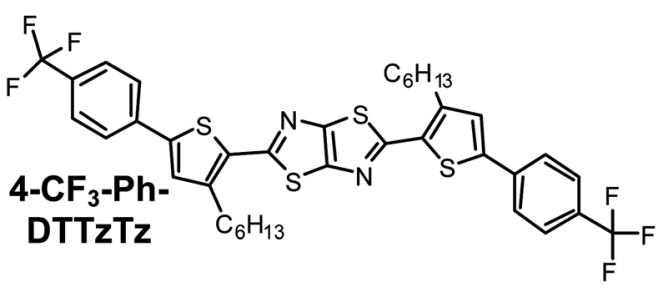
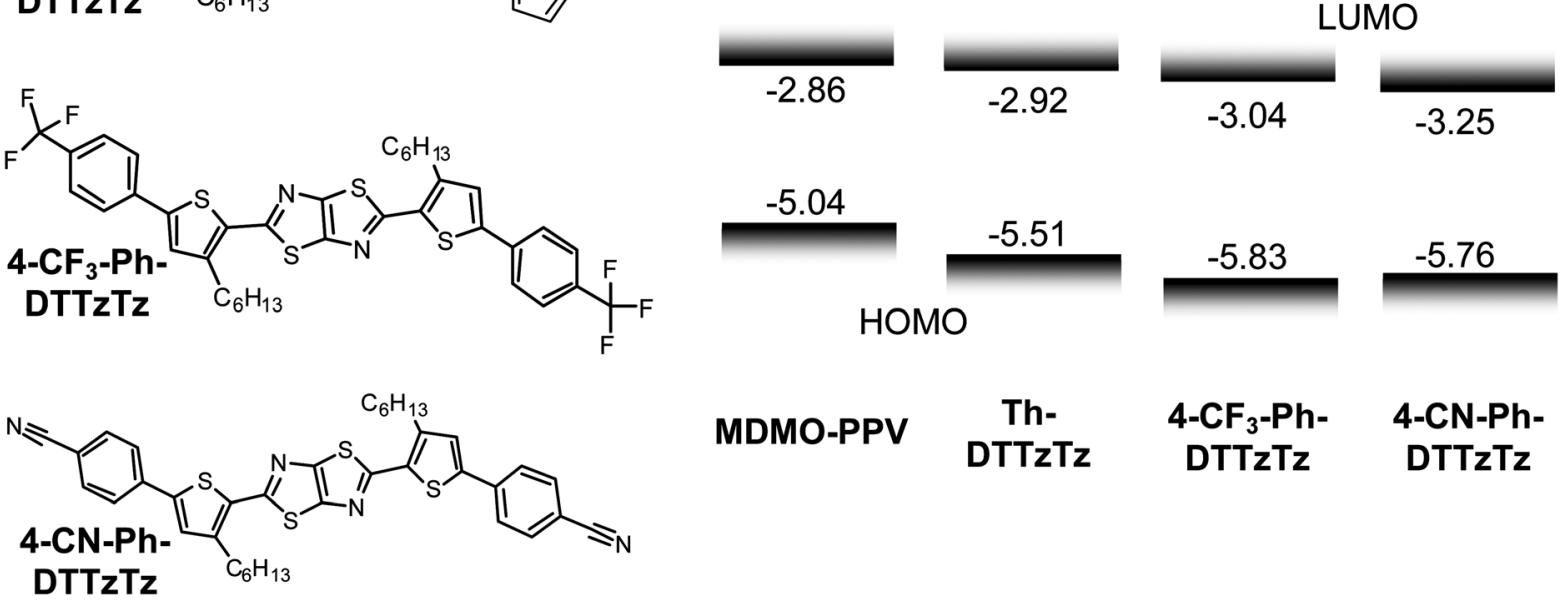

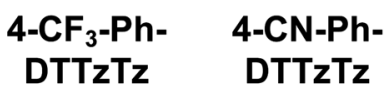

Fig. 1 Molecular structures of the DTTzTz compounds and alignment of the energy levels according to cyclic voltammetry data (Table 1), correlating well with calculated values of the HOMO and LUMO energies. ${ }^{9,10}$ 
poly[2-methoxy-5-(3'-7'-dimethyloctyloxy)-1,4-phenylene vinylene] (MDMO-PPV), in which a small driving force for charge separation could be expected, systematically increasing in the series of DTTzTz compounds.

\section{Materials and methods}

\section{Sample preparation}

The synthesis of 2,5-bis(4-hexyl-2,2'-bithiophene-5-yl)thiazolo[5,4- $d]$ thiazole (Th-DTTzTz), 2,5-bis( $3^{\prime}$-hexyl-5'-[4-(trifluoromethyl)phenyl]thiophen-2'-yl)thiazolo[5,4- $d$ ] thiazole (4-CF $\mathbf{-}-\mathbf{P h}$ DTTzTz) and $4,4^{\prime}$-[5,5'-(thiazolo[5,4- $\left.d\right]$ thiazole-2,5-diyl)-bis(4-hexylthiophene-5,2-diyl)]dibenzonitrile (4-CN-Ph-DTTzTz) has been reported elsewhere. ${ }^{9,10}$ MDMO-PPV was obtained from Covion $\left(M_{\mathrm{w}}=716 \mathrm{~kg} \mathrm{~mol}^{-1}, \mathrm{PD}=6.5\right)$. Solutions were prepared in chloroform $\left(\mathrm{CHCl}_{3}\right.$, Acros Organics, $\left.99.9 \%\right)$.

For optical characterization, thin films were prepared by spin-coating (speed: $1000 \mathrm{rpm}, 60 \mathrm{~s}$ spin time) from a $\mathrm{CHCl}_{3}$ solution $\left(10 \mathrm{mg} \mathrm{mL}^{-1}\right.$, total weight of DTTzTz and polymer over solvent volume). Besides the pristine MDMO-PPV polymer sample, a set of 3 samples was prepared for each DTTzTz material, namely spin-coated films of the pristine molecules and of two MDMO-PPV : 4-CN-Ph-DTTzTz blends of $4: 1$ and $1: 1$ weight ratio, respectively. EPR samples were produced from the same solutions by drop casting of a well defined volume onto a glass substrate (to ensure equal amounts) and collecting the materials from the substrate to transfer them into the quartz sample tubes. The thickness of the spin-coated films was measured using a Dektak profilometer $\left({ }^{3} \mathrm{ST}\right.$, Sloan/Veeco Instr.). The values (see ESI, $\dagger$ Table S1) for the pure DTTzTz films were the lowest (in the range 53-84 nm), while the pure MDMO-PPV reference film was the thickest $(256 \mathrm{~nm})$, and the 50 : 50 blends come in between (169-228 nm), corresponding to an expected increased viscosity with increased polymer concentration.

\section{Optical spectroscopy}

UV-visible absorption spectroscopy measurements were performed on a Varian CARY 500 spectrophotometer. A Varian Cary Eclipse fluorometer was used for the measurement of the PL spectra which were corrected for wavelength dependent sensitivity of the instrument. Time-resolved PL (TR-PL) spectra were obtained by streak camera detection (50 ps resolution) after excitation with $400 \mathrm{~nm}$ laser pulses (repetition rate $82 \mathrm{MHz}$, pulse width $<2 \mathrm{ps}$ ) from the frequency-doubled output of a mode-locked Ti-sapphire laser (Spectra Physics).

PIA was performed by detection of the probe light of a halogen lamp transmitted through the spin-coated films under excitation at $532 \mathrm{~nm}$ using a frequency-doubled CW Nd:YAG laser beam (10 $\mathrm{mW}$ spread over a $3 \mathrm{~mm}$-aperture) that was on/off modulated by means of an acousto-optic device. The PIA spectrum is obtained as the lock-in detected modulated signal normalised by the transmitted probe intensity at each wavelength. A digital storage oscilloscope allows registration of the time-dependent signal after switch-on and switch-off of laser excitation. High-energy photons were eliminated from the probe beam using a $560 \mathrm{~nm}$ long-wavelength pass (LWP) filter before the sample to minimize photodegradation.
The collected light was passed through a $580 \mathrm{~nm}$ LWP filter to stop laser scattering - and an $f=30 \mathrm{~cm}$-monochromator before detection by a silicon photodiode. The signals detected without a probe beam were subtracted to correct for background and to eliminate PL. The PIA measurements were performed at $T=80 \mathrm{~K}$ and up to room temperature (RT) in a cryostat under vacuum in order to avoid photo-oxidation of the materials.

\section{EPR spectroscopy}

X-band continuous-wave (CW) EPR experiments were performed using a Bruker ESP300E spectrometer, at microwave frequency $\sim 9.4 \mathrm{GHz}$, equipped with a liquid helium flow cryostat (Oxford Instr., Inc.). The presented CW-EPR spectra are recorded using $0.1 \mathrm{mT}$ field modulation amplitude, $100 \mathrm{kHz}$ modulation frequency, and $5 \mathrm{~mW}$ microwave power. W-band ESE (electron spin echo) detected EPR experiments were performed on a Bruker Elexsys E680 spectrometer (microwave frequency $\sim 94 \mathrm{GHz}$ ), equipped with a continuous flow cryostat and a superconductive magnet from Oxford Instr., Inc. A $\pi / 2-\tau-\pi-\tau$-echo pulse sequence was used, with pulse lengths $t_{\pi}=240 \mathrm{~ns}, t_{\pi / 2}=120 \mathrm{~ns}$ and pulse interval $\tau=700 \mathrm{~ns}$. All EPR experiments were performed before and during illumination with the $488 \mathrm{~nm}(2.54 \mathrm{eV})$ output of an Ar ion laser with $25 \mathrm{~mW}$ power (Spectra Physics). The X- and W-band LI-EPR spectra are all presented as difference spectra (light-minus-dark) in order to determine the light-induced components. To allow direct comparison of the EPR spectra, the spectra are shown rescaled to the same microwave frequency of 9.4 and $94 \mathrm{GHz}$ for X- and W-band measurements, respectively. The created radicals decay fast at $\mathrm{RT}$, therefore all the EPR measurements were performed at $100 \mathrm{~K}$.

\section{Devices preparation and characterisation}

Devices with blends of MDMO-PPV and 4-CN-Ph-DTTzTz as an active layer were prepared using standard procedures for bulk heterojunction solar cells with a layer sequence glass substrate/ITO/ PEDOT-PSS/blend/Ca/Al. Tuning of the processing conditions (concentration, solvent, blend ratio and spin-coating parameters) led to a power conversion efficiency of $0.1 \%$ in the best case $(1: 1$ blend, layer thickness $\sim 50 \mathrm{~nm}$, spin-coating from a toluene solution), combining a large open circuit voltage $\left(V_{\mathrm{OC}}=1.39 \mathrm{~V}\right)$ with low values for both the short-circuit current $\left(I_{\mathrm{SC}}=\right.$ $\left.0.29 \mathrm{~mA} \mathrm{~W}^{-1}\right)$ and the fill factor $(\mathrm{FF}=0.24)$. The spectral dependence of the external quantum efficiency (EQE) of such devices was measured by means of Fourier transform photocurrent spectroscopy (FTPS). ${ }^{27}$

\section{Results}

\section{Optical absorption and external quantum efficiency}

The optical absorption spectra of the pure MDMO-PPV polymer and the thiazolo[5,4- $d$ ]thiazole derivatives were measured both in chloroform solution (ESI, $\dagger$ Fig. S1, solid curves) and in thin films (Fig. 2A, dashed curves). A summary of the optical data for the pure compounds is listed in Table 1, including the optical bandgaps derived from the low energy onsets of the absorption bands. Complementary information on the HOMO and LUMO energy levels and the resulting bandgaps as derived 
for the 4 compounds from cyclic voltammetry $(\mathrm{CV})$ in $\mathrm{CH}_{3} \mathrm{CN}$ solution is also listed. ${ }^{9,10}$ Among the workhorse donor polymers for BHJ solar cells, MDMO-PPV was chosen as a partner to the DTTzTz molecules because of the high LUMO level increasing the chances for efficient CT. The tendency for crystallization in the pure DTTzTz spin-coated films resulted in long-wavelength tails due to diffuse scattering. Similar absorption efficiencies were found for the four pure compounds in solution. The spectra in the films were quite strongly altered in intensity, position as well as shape. The film thickness (see ESI, $\dagger$ Table S1) is the first and evident factor correlating with the overall absorption intensity and it was found to increase with increasing polymer content.

Further, a significant red shift was found for the $\lambda_{\text {onset }}$ of the pure DTTzTz molecules when going from solution to the solid state (in thin film), corresponding to a decrease in bandgap, which may be attributed to a more planar structure in the solid state (resulting from $\pi-\pi$ stacking) and to electronic interactions between neighbouring molecules. This decrease is somewhat less important for Th-DTTzTz than for the derivatives substituted with phenyl entities, i.e. 4-CN-Ph-DTTzTz and 4-CF3-Ph-DTTzTz. As a result, Th-DTTzTz possesses the largest bandgap of the DTTzTz molecules in the solid state, while this was the smallest in solution (see Table 1). While in solution for all DTTzTz derivatives the HOMO-LUMO transition is at markedly higher energy than for the MDMO-PPV polymer (ESI, $\dagger$ Fig. S1, solid curves), there is a large overlap of the absorption bands of the pure DTTzTz chromophores and the polymer in the films (Fig. 2A, dashed curves) and correspondingly the values of the DTTzTz bandgaps are only $0.05-0.13 \mathrm{eV}$ higher than that of the polymer (Table 1). The vibrational structure is hardly resolved in the absorption spectra in solution (ESI, $\dagger$ Fig. S1, solid curves), but for all of the DTTzTz molecules it becomes more pronounced in the spin-cast films (Fig. 2A, dashed curves). The first few vibrational components are observed at very similar positions for the three molecules, but the intensity distribution is different for $\mathbf{4}-\mathbf{C F}_{\mathbf{3}}-\mathbf{P h}-\mathbf{D T T z T z}$ compared to the other compounds, with the intensity reaching a maximum at higher energy on the third instead of the second vibrational component, pointing to a stronger electron-vibrational coupling.

The absorption spectra of the $1: 1$ blend polymer : DTTzTz films are also shown in Fig. 2A (solid curves). Inspection of the curves shows that they cannot be reproduced by a linear combination of the spectra of the pure polymer and DTTzTz films, which is most easily seen in the tail region of the bands where the blends have higher absorption than each of the individual components. The shoulder is most obvious in the case of the blend with 4-CN-Ph-DTTzTz which indicates a significant intermolecular electronic interaction between the donor and acceptor compounds in the ground state. From the absorption onset, energy differences of $0.06 \mathrm{eV}, 0.08 \mathrm{eV}$ and $0.12 \mathrm{eV}$ relative to the MDMO-PPV film could be estimated, increasing in the series Th-, $\mathbf{4}-\mathbf{C F}_{\mathbf{3}}-\mathbf{P h}$ - and 4-CN-Ph-DTTzTz.

The decrease of bandgap in the blend materials is confirmed by FTPS measurements (see Fig. 2B) of a BHJ device based on a MDMO-PPV : 4-CN-Ph-DTTzTz $1: 1$ blend (solid curve) compared to devices with the pure materials as the active layer (dashed curves). The EQE spectrum of the BHJ device reveals a significant shoulder in the spectral response of the EQE in the region just below the polymer bandgap. For the three devices, the onset of the EQE corresponds precisely to the one determined in optical absorption for each of the corresponding spin-cast films. The EQE measurements demonstrate that the band edge optical transitions in the blends generate charge carriers that can contribute to the photocurrent in the solar cell device. The nature of these band edge transitions is further discussed below.

\section{Photoluminescence quenching}

PL measurements of the pure compounds were performed in solution (ESI, $\dagger$ Fig. S1, symbols) and on spin-cast films

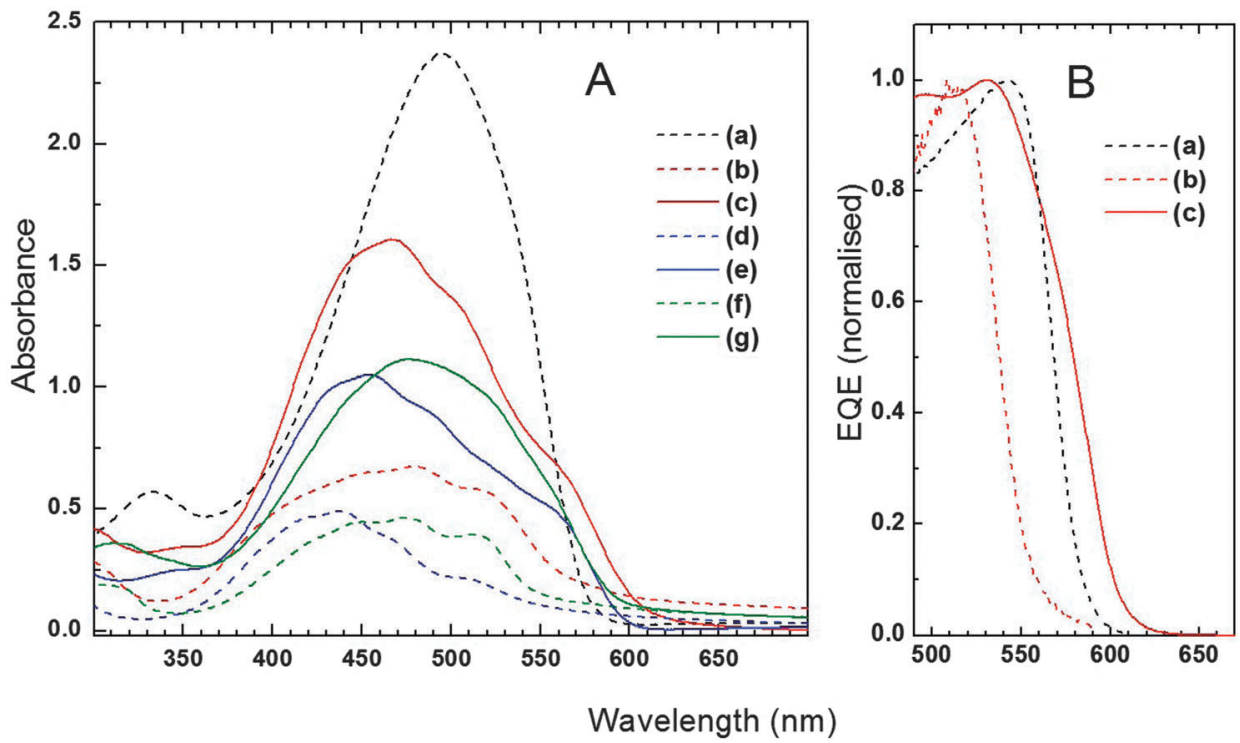

Fig. 2 (A) UV-vis absorption spectra of spin-cast films (a) MDMO-PPV, (b) 4-CN-Ph-DTTzTz, (c) $1: 1$ blend of MDMO-PPV : 4-CN-Ph-

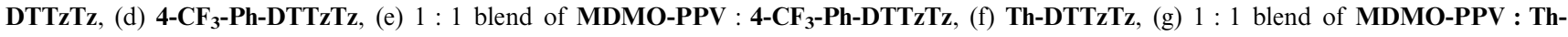
DTTzTz. Graph (B) shows the EQE spectra of devices with active layers consisting of the corresponding materials (a-c). 
Table 1 Optical bandgap data $\lambda_{\text {onset }}$ and $E_{\mathrm{g}}(\mathrm{opt})$ for the DTTzTz materials measured both in pristine and blend spin-coated films, compared to those measured in $\mathrm{CHCl}_{3}$ solution. $\mathrm{HOMO}$ and LUMO energies and derived bandgap $E_{\mathrm{g}}(\mathrm{CV})$ from cyclic voltammetry $(\mathrm{CV})$ measurements in MeCN. ${ }^{9,10}$ Data for MDMO-PPV are included for comparison

\begin{tabular}{|c|c|c|c|c|c|c|c|c|c|}
\hline & \multicolumn{2}{|c|}{ Pristine films } & \multicolumn{2}{|c|}{ MDMO-PPV:DTTzTz films } & \multicolumn{2}{|c|}{$\mathrm{CHCl}_{3}$ solution } & \multicolumn{3}{|c|}{ Cyclic voltammetry (in $\mathrm{MeCN}$ ) } \\
\hline & $\lambda_{\text {onset }}{ }^{a} / \mathrm{nm}$ & $E_{\mathrm{g}}(\mathrm{opt})^{a} / \mathrm{eV}$ & $\lambda_{\text {onset }}{ }^{a} / \mathrm{nm}$ & $E_{\mathrm{g}}(\mathrm{opt})^{a} / \mathrm{eV}$ & $\lambda_{\text {onset }}{ }^{a} / \mathrm{nm}$ & $E_{\mathrm{g}}(\mathrm{opt})^{a} / \mathrm{eV}$ & $\mathrm{HOMO} / \mathrm{eV}$ & $\mathrm{LUMO} / \mathrm{eV}$ & $E_{\mathrm{g}}(\mathrm{CV}) / \mathrm{eV}$ \\
\hline 4-CN-Ph- & 559 & $2.22^{b}$ & 607 & 2.04 & 502 & 2.47 & -5.76 & -3.25 & 2.51 \\
\hline 4-CF3-Ph- & 560 & $2.21^{b}$ & 597 & 2.08 & 489 & 2.54 & -5.83 & -3.04 & 2.79 \\
\hline Th- & 541 & $2.29^{b}$ & 591 & 2.10 & 505 & 2.46 & -5.51 & -2.92 & 2.59 \\
\hline MDMO-PPV & 574 & 2.16 & - & - & 551 & 2.25 & -5.04 & -2.86 & 2.18 \\
\hline
\end{tabular}

${ }^{a}$ Onset of optical absorption $\lambda_{\text {onset }}$ and optical bandgap $E_{\mathrm{g}}(\mathrm{opt})$ determined from the crossing of the tangent to the long-wavelength tail of the absorption band with the baseline. ${ }^{b}$ Uncertainty of $\pm 0.02 \mathrm{eV}$, due to the presence of a long wavelength tail ascribed to scattering from the spincoated films of the small molecules.

(Fig. 3, dashed curves). As for the absorption spectra, the emission of the DTTzTz compounds in the films is red-shifted compared to the corresponding solutions leading to a nearcomplete overlap of the DTTzTz PL spectra with that of MDMO-PPV. While the onset of the absorption is still at slightly higher energies than for the polymer, the onset of emission is even more red-shifted and ends up at lower energy than for MDMO-PPV, pointing to additional configurational relaxation in the DTTzTz films. The important overlap in both absorption and emission of DTTzTz and polymer spectra hampers the use of selective spectroscopy and makes it hard to determine the origin of the PL in the blends.

The quenching of the PL intensity was examined as an indicator for the efficiency of CT between the MDMO-PPV polymer (as a donor) and the DTTzTz materials (as acceptors), competing with the radiative decay process. This can be monitored by the decrease in intensity (from time-integrated PL spectra) as well as by the increase in decay rates (from TR-PL).

In Fig. 3, one can compare the emission spectra from the pristine polymer and the pure acceptor materials (dashed curves) with those from $1: 1$ blends (solid curves). In this figure, the emission spectra are normalised to the number of absorbed photons, on the basis of the absorbance of the films measured at the excitation wavelength of $474 \mathrm{~nm}$. It should be

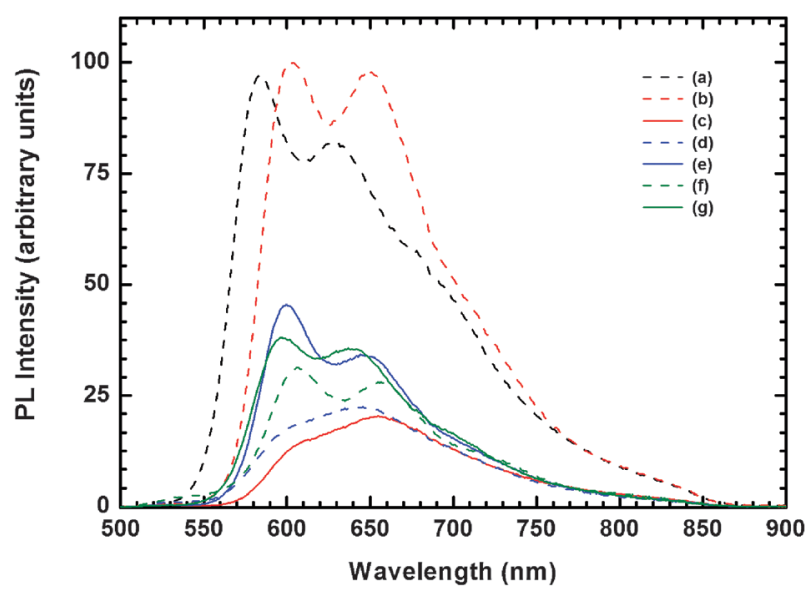

Fig. 3 PL spectra (normalized for the number of photons absorbed at $474 \mathrm{~nm}$ ) of spin-cast films of (a) MDMO-PPV, (b) 4-CN-Ph-DTTzTz, (c) $1: 1$ blend of MDMO-PPV : 4-CN-Ph-DTTzTz, (d) 4-CF 3 -Ph-DTTzTz, (e) $1: 1$ blend of MDMO-PPV : 4-CF - Ph-DTTzTz, (f) Th-DTTzTz, (g) $1: 1$ blend of MDMO-PPV : Th-DTTzTz. kept in mind that both the MDMO-PPV polymer and the DTTzTz compounds exhibit quite efficient PL, unlike the well-studied polymer:fullerene blends in which the fullerene emission is usually very weak. Among the pure DTTzTz small molecules, the PL efficiency of 4-CN-Ph-DTTzTz is comparable to that of pristine MDMO-PPV and significantly higher than for 4-CF3-Ph-DTTzTz and Th-DTTzTz, which points to more efficient non-radiative decay pathways in the latter compounds in the solid state.

In the case of 4-CN-Ph-DTTzTz, a clear quenching of PL emission (reduction to $<15 \%$ ) is observed when going from either the pristine polymer or the pure DTTzTz material to the blend film, and this can be considered as an indication of efficient CT. For 4-CF $\mathbf{3}-\mathbf{P h}-\mathbf{D T T z T z}$ and Th-DTTzTz, the results are not decisive for PL quenching: the total PL is reduced by hardly more than a factor 2 compared to the pristine polymer and the emission is in both cases stronger than for the pure DTTzTz films (while each component represents half of the blend material). It seems most probable that the emission from these two blends originates to a large extent from the polymer fraction, and additional indication for this is discussed below, derived from the TR-PL measurements. It should be noted here, however, that the spectra are shifted to markedly lower energy compared to the MDMO-PPV film. It seems plausible that in such intimate mixtures of compounds the exciton recombination occurs after migration and trapping at states of lower energy induced by heteromolecular interactions between polymer and acceptor molecules.

Fig. 4A shows the TR-PL spectra (normalized to the number of absorbed photons at $400 \mathrm{~nm}$ ) of spin-coated films of the pristine polymer and the DTTzTz derivatives (dashed curves) as well as of the $1: 1$ polymer : DTTzTz blends (solid curves), while Fig. 4B shows these decays normalized to peak intensity and displayed on a semi-log scale. The two types of graphs emphasize more on the aspect of relative intensity and of decay rate, respectively. Two of the pure DTTzTz films (4-CF ${ }_{3}$-Ph-DTTzTz and 4-CN-Ph-DTTzTz) display a near to single exponential decay with a time constant close to $1 \mathrm{~ns}$, while the Th-DTTzTz compound displays a much faster and non-exponential decay with a characteristic time (1/e value) of $\sim 200$ ps, quite similar to the pristine MDMO-PPV film. While the PL decay in MDMO-PPV is much faster than in 4-CN-Ph-DTTzTz, these compounds show comparable total emission efficiencies (Fig. 3, dashed curves), markedly higher than the two remaining materials. 

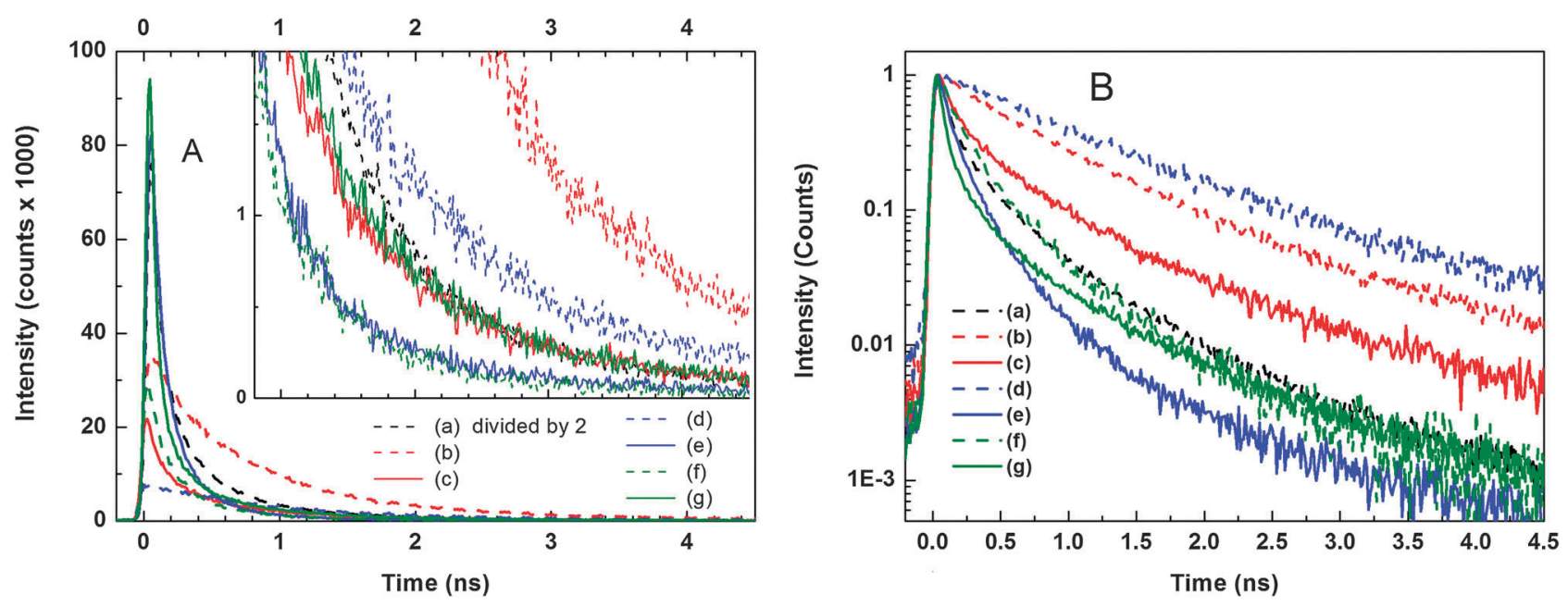

Fig. 4 TRPL spectra of (a) MDMO-PPV (intensity in A divided by 2), (b) 4-CN-Ph-DTTzTz, (c) $1: 1$ blend of MDMO-PPV : 4-CN-PhDTTzTz, (d) 4-CF - Ph-DTTzTz, (e) $1: 1$ blend of MDMO-PPV : 4-CF - -Ph-DTTzTz, (f) Th-DTTzTz, (g) $1: 1$ blend of MDMO-PPV : ThDTTzTz. Graph (A) shows the data on a linear scale within the inset the tail of the decays vertically expanded, whereas graph (B) shows the normalized data on a semilog scale, emphasizing intensity and decay rate information, respectively.

For MDMO-PPV : 4-CN-Ph-DTTzTz (1 : 1), two contributions can be discerned in the decay curve in TR-PL, i.e. a fast and a slow component, with characteristic times that are similar to the ones of the pristine polymer and the pure DTTzTz films, respectively. However, both these components are strongly reduced in intensity compared to the pure films, which is well in line with the PL quenching derived from the spectral measurements.

The situation is quite different for the two other blends. Clearly, the intensity increases relative to the pure DTTzTz film observed in the PL spectra (Fig. 3) is corresponding here to a strong enhancement of a fast component in the decay curves with decay time similar to but smaller than that in pure MDMO-PPV (see also normalised curves e and g in Fig. 4B), with a peak intensity close to half of that in the pure polymer. This increased fast component can be ascribed to PL from the MDMO-PPV polymer, which constitutes half of the material in these blends, and consequently would be hardly quenched in these cases. Furthermore, at longer times, the decay curve of the blend with Th-DTTzTz never goes below that of the pure molecule. This is different for the blend with 4-CF $\mathbf{- P h}-\mathbf{D T T z T z}$ where the tail of the decay after $\sim 0.7$ ns lies below that of the pure material, which can be considered as partial quenching of the PL from the DTTzTz component. For these two compounds one cannot draw definite conclusions from the PL data alone, but it will be shown below that these observations correlate well with those from LI-EPR and PIA experiments.

\section{Polaron detection by electron paramagnetic resonance}

As a consequence of CT one expects the production of positive and negative molecular radicals, also called polarons in the context of organic semiconductors, in the donor and acceptor materials, respectively. Direct evidence of radical production can be provided by EPR spectroscopy.

As a reference measurement, LI-EPR of the pristine DTTzTz powders and of a pristine MDMO-PPV polymer film was measured (Fig. 5A, curves a and b, respectively). No light-induced signals were observed in the pure DTTzTz samples, while the MDMO-PPV film showed a weak signal. This type of weak LI-EPR signal in pure PPV compounds has been reported previously, ${ }^{20,31}$ and is ascribed to material defects acting as traps for the photogenerated carriers. The LI-EPR signals in the $1: 1$ polymer : DTTzTz blends were found to be significantly more intense than in the pristine MDMO-PPV film (Fig. 5A, curves c-e; the spectra are shown in overlay in ESI, $\uparrow$ Fig. S3A), and the LI-EPR intensity was found to follow the order 4-CN-Ph-DTTzTz > 4-CF3-PhDTTzTz > Th-DTTzTz. This was observed for different batches and the same trend was also found for polymer : DTTzTz 4 : 1 films (ESI, $\dagger$ Fig. S3B). The LI-EPR signal in the latter films was found to be systematically smaller than in the corresponding 1: 1 blends (ESI, $\dagger$ Fig. S3C, for the case 4-CN-Ph-). These observations clearly demonstrate that radicals are formed upon illumination of the blends and that this radical formation depends on the relative DTTzTz amount. Hence, CT is shown to occur between the polymer and the small molecules. From the position of the HOMO-LUMO levels determined for the thiazolo[5,4- $d$ ] thiazoles and the polymer in solution (see Fig. 1 and Table 1), it seems likely that electron transfer from the polymers to the DTTzTz semiconductors has occurred upon photoexcitation, and a positive and negative polaron would hence be expected in these compounds, respectively. Since in X-band EPR only a single resonance line at $g=$ 2.0024 is observed, which can originate from one or more radical species, we performed LI-EPR experiments at higher microwave frequency (W-band, at $\sim 94 \mathrm{GHz}$ ). Fig. 6 shows the light-induced W-band ESE-detected EPR signal obtained from a drop-cast film of the MDMO-PPV : 4-CN-Ph-DTTzTz $1: 1$ blend, which displayed the highest CT efficiency according to the corresponding X-band measurements reported in Fig. 5. Because of the higher resolution at $\mathrm{W}$-band microwave frequencies, the LI-EPR spectrum now reveals distinct features. For comparison, the previously reported ${ }^{32} \mathrm{~W}$-band ESE-detected EPR 

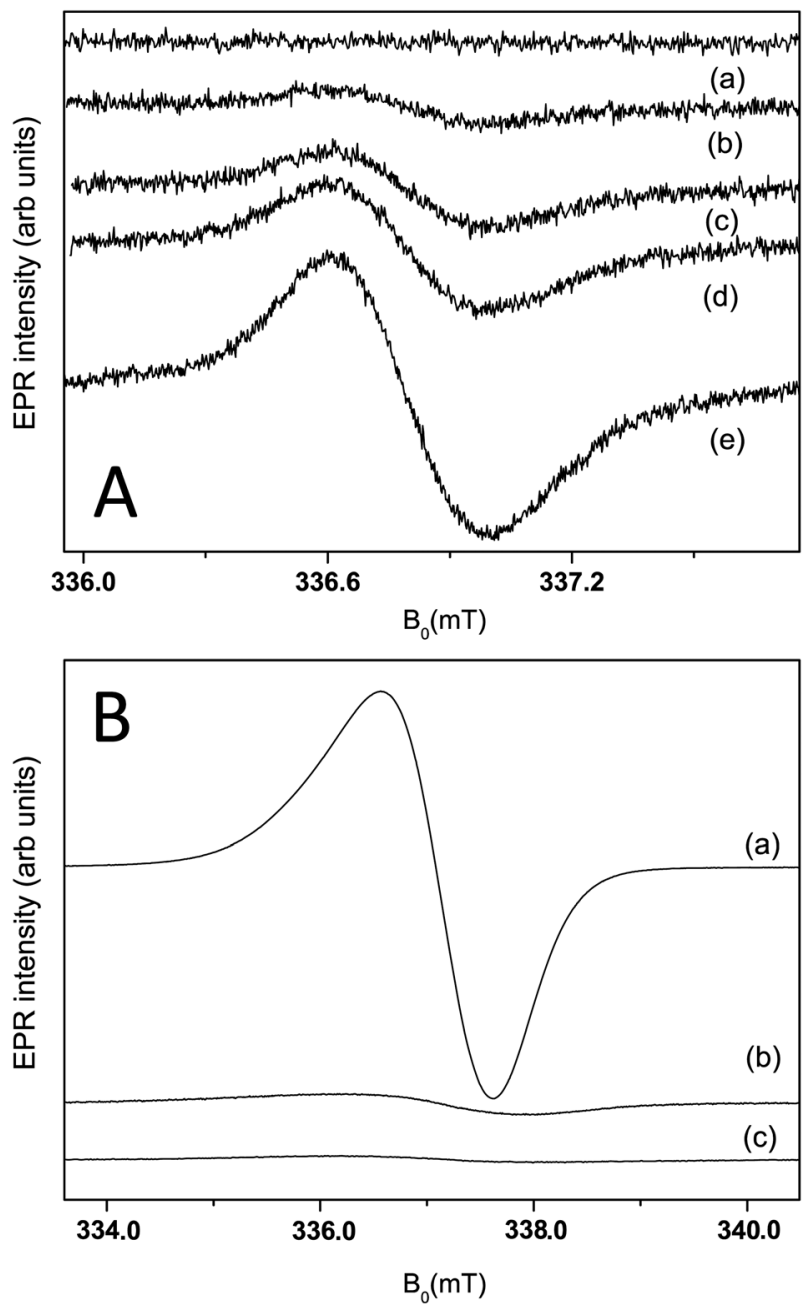

Fig. 5 (A) LI-EPR spectra (a) in 4-CN-Ph-DTTzTz, (b) in MDMO-PPV and in the $1: 1$ blends of (c) MDMO-PPV : Th-DTTzTz, (d) MDMO-PPV : 4-CF 3 -Ph-DTTzTz and (e) MDMO-PPV : 4-CN-PhDTTzTz (all in drop cast films except for (a) which was measured in a powder sample); (B) positive polaron EPR spectra induced by $\mathrm{I}_{2}$ doping of pure DTTzTz powders: (a) Th-DTTzTz, (b) $\mathbf{4}-\mathbf{C F}_{\mathbf{3}}-\mathbf{P h}-$ DTTzTz and (c) 4-CN-Ph-DTTzTz (CW X-band EPR at $T=$ $100 \mathrm{~K}$; difference spectra before and during illumination (in A) and before and after $\mathrm{I}_{2}$ doping (in B)).

spectrum of the positive radical or polaron obtained by chemical doping of MDMO-PPV with $\mathrm{I}_{2}$ is shown. The signal of this positive radical is clearly also present in the LI-EPR spectrum of the MDMO-PPV : 4-CN-Ph-DTTzTz (1:1) blend (signal marked with asterisk in Fig. 6), together with a second feature at a higher $g$ value (signal marked with \# centered at $g=2.0045$ in Fig. 6). Hence, the latter signal can be ascribed to the negative DTTzTz radical, analogous to the negative fullerene radical observed in LI-EPR of polymer: fullerene blends. ${ }^{19,20}$ These observations provide a clear proof of CT between the polymer and 4-CN-Ph-DTTzTz in these blends. Note that the excitation wavelength $(488 \mathrm{~nm})$ was chosen such as to excite both the MDMO-PPV and the small molecule (see also Fig. 2), and that attempts to vary the wavelength for selective excitation of either the polymer or DTTzTz component were unfortunately not successful.

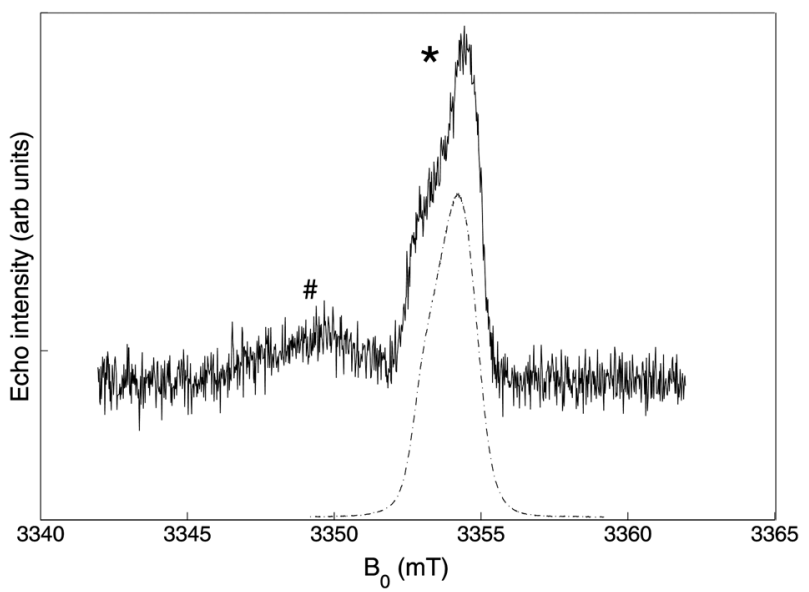

Fig. 6 W-band light-induced ESE-detected EPR spectrum of a $1: 1$ MDMO-PPV : 4-CN-Ph-DTTzTz blend (solid line) compared to the ESE-detected EPR spectrum of $\mathrm{I}_{2}$-doped MDMO-PPV (dash dotted line). The asterisk marks the signal of the positive MDMO-PPV polaron, whereas the \# mark indicates the signal of the negative 4CN-Ph-DTTzTz radical.

As a complementary experiment, $\mathrm{I}_{2}$ doping was applied to evaluate the efficiency of positive polaron production in the pure DTTzTz materials. As shown in Fig. 5B, the EPR signal is very strong in Th-DTTzTz, much smaller in $\mathbf{4 - C F}_{\mathbf{3}}-\mathbf{P h}-\mathrm{DTTzTz}$, and hardly visible in 4-CN-Ph-DTTzTz, which is interestingly anti-correlated with the LI-EPR in the corresponding blends. This indicates that the acceptor level of the dopant $I_{2}$ lies below the HOMO of Th-DTTzTz, but not sufficiently deep for efficient oxidation of the other molecules. This is again consistent with the much lower position of the HOMO level of $4-\mathbf{C F}_{\mathbf{3}}-\mathbf{P h}-\mathbf{D T T z T z}$ or 4-CN-Ph-DTTzTz relative to Th-DTTzTz, as determined by $\mathrm{CV}$ in solution (see Fig. 1 and Table 1).

\section{Positive polaron detection by photoinduced absorption}

As an additional confirmation of the CT, a clear PIA signal was obtained at $T=95 \mathrm{~K}$ in spin-coated films of the $1: 1$ blends of MDMO-PPV with the DTTzTz molecules, as shown in Fig. 7 (curves a-c). The band at $930 \mathrm{~nm}$ can be attributed to the positive polaron in the polymer, as reported previously for MDMO-PPV:PCBM blends. ${ }^{23}$ Again, the intensity is highest for the blend with 4-CN-Ph-DTTzTz as the acceptor, decreasing

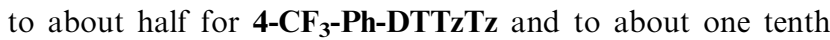
for Th-DTTzTz, which is consistent with the LI-EPR results measured at $T=100 \mathrm{~K}$.

It is, however, important to notice that the polaron band disappears in these blends when the PIA is performed at RT. For the case with the strongest polaron PIA, the blend with 4-CN-Ph-DTTzTz, the time dependence of the signal is shown at $T=95 \mathrm{~K}$ and at $229 \mathrm{~K}$ in the inset of Fig. 7. It shows a characteristic time (1/e value) for decay of the polaron population of $\tau_{\downarrow}=85 \mu$ s measured after switch-off of the laser excitation. The decay time is found to decrease by about a factor 3 already at $T=230 \mathrm{~K}, \tau_{\downarrow}=24 \mu \mathrm{s}$, with concomitant decrease in the PIA amplitude. The increase in the rate of charge carrier recombination indicates that this process is 


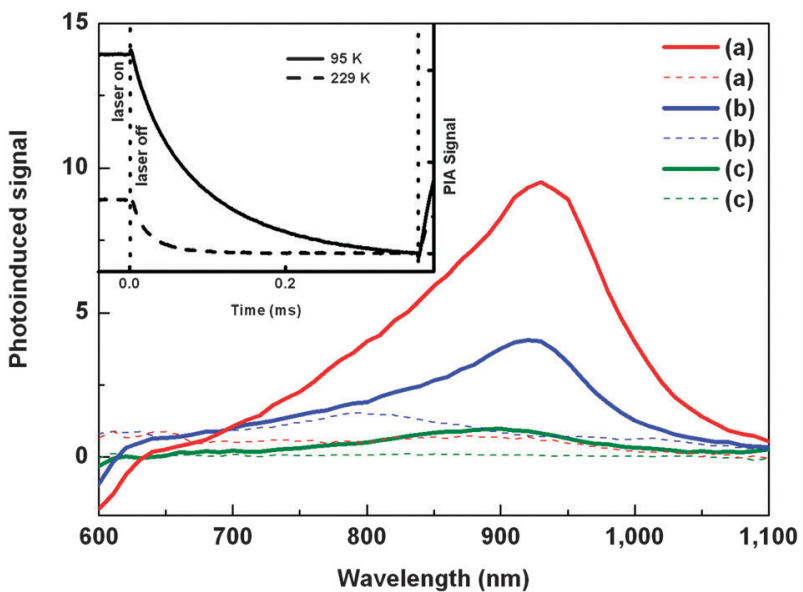

Fig. 7 PIA spectra at $T=95 \mathrm{~K}$ (solid curves) and RT (dashed curves) of spin-coated films of $1: 1$ blends of (a) MDMO-PPV : 4-CN-Ph-DTTzTz, (b) MDMO-PPV : 4-CF - -Ph-DTTzTz, and (c) MDMO-PPV : Th-DTTzTz. The inset shows the time dependence of the PIA signal after switching off the $532 \mathrm{~nm}$ laser excitation modulated at $1.37 \mathrm{kHz}$.

thermally activated over a relatively low barrier. We have compared this under the same measuring conditions with a spin-coated film of an MDMO-PPV : PCBM 1:1 blend prepared with the same parameters (solvent, concentrations, spin coating,...). This is displaying a much slower PIA decay of $\tau_{\downarrow}=660 \mu$ s at $T=90 \mathrm{~K}$, which in this blend is found to persist up to RT, with a decay time of $\tau_{\downarrow}=60 \mu$ s still comparable to that in the blend with 4-CN-Ph-DTTzTz at the lowest temperature (see ESI, $\uparrow$ Fig. S4). It is worth mentioning here that the detected PIA at the lowest temperatures is markedly stronger in the blend with 4-CN-Ph-DTTzTz than in the one with PCBM, while the latter is known as a very efficient BHJ OSC material. Here, one should take into account the lower number of absorbed photons in the case of MDMO-PPV:PCBM, a factor $\sim 2$ as derived from the absorbances at $532 \mathrm{~nm}$, but even then the low temperature PIA signal is about five times higher in the MDMO-PPV:4-CN-Ph-DTTzTz film. However, upon warming the reduction in PIA signal for PCBM as acceptor is quite limited, and at RT it is clearly stronger than that for the case of 4-CN-Ph-DTTzTz (see ESI, $\dagger$ Fig. S4).

\section{Discussion}

The PL quenching, LI-EPR and PIA results demonstrate that optically induced CT is hardly detectable in the MDMO-PPV: Th-DTTzTz blend, while it is clearly observed in MDMO-PPV: 4-CF - Ph-DTTzTz and most effective in the MDMO-PPV:4-CNPh-DTTzTz blends. These results correlate well with the increasing electron-acceptor character within this series of DTTzTz compounds, as indicated by the HOMO and LUMO level positions available from CV in solution (Fig. 1 and Table 1), which were also underpinned by density functional theory calculations of the electronic properties. ${ }^{9,10}$ The CT to a reference donor polymer demonstrated for 4-CN-Ph-DTTzTz, in combination with the high charge mobility expected in this type of thiophene-based derivatives, is promising for the application of related DTTzTz materials as acceptors in D-A blends for 3D BHJ solar cells.
The CT process is a net electron transfer from polymer to DTTzTz which happens upon dissociation of excitons, but these could be produced by photon absorption in the donor material as well as in the acceptor material. The two possibilities have indeed been previously demonstrated by photocurrent spectroscopy in polymer:fullerene BHJ devices, in which however the absorption in the donor polymer yields the main contribution. The exciton in the donor is providing an electron in the donor LUMO that can be transferred to the acceptor LUMO, while the exciton in the acceptor material consists of a hole in the acceptor HOMO that can be filled by an electron transferred from the donor HOMO (or in semiconductor terms: a hole could be transferred from acceptor to donor), both processes resulting in the same charge separated state. Unfortunately, we have not been able to study preferential optical excitation in either of the components of the studied blends because of the strong mutual overlap of their optical spectra in the solid state (see Fig. 2 and 3).

In solution, important differences in energy are found between the HOMO level in MDMO-PPV and in the DTTzTz derivatives, which amount to $0.72 \mathrm{eV}$ for 4-CN-Ph-DTTzTz, $0.79 \mathrm{eV}$ for $\mathbf{4}-\mathbf{C F}_{\mathbf{3}}-\mathbf{P h}-\mathbf{D T T z T z}$ and $0.47 \mathrm{eV}$ for Th-DTTzTz. Energetically the requirement for 'hole' transfer would seem to be fulfilled in all cases. It is therefore tempting to ascribe the observed trend in CT efficiency to the LUMO alignment with decreasing differences relative to MDMO-PPV in the series

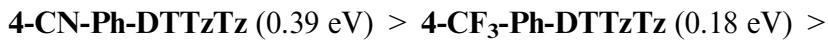
Th-DTTzTz $(0.06 \mathrm{eV})$. However, this turns out to be much less evident when one considers the data in spin-coated films. Indeed, the band-gaps derived from optical absorption spectra are hardly higher for the small molecules in the solid state than for MDMO-PPV, and thus alignment between the LUMOs of polymer and small molecules occurs necessarily in parallel with alignment between the HOMO levels. Therefore, energy constraints for electron and for hole transfer will be coupled: increasing acceptor character will lead to improvements in both HOMO and LUMO alignments, and it is not straightforward to pinpoint the observed correlation to be either donor-toacceptor electron transfer or acceptor-to-donor hole transfer. The TR-PL data seem to indicate that the PL quenching occurs in both the polymer and DTTzTz components in the blend with 4-CN-Ph-DTTzTz, which can be ascribed to two-ways CT after excitation in either of the donor and acceptor compounds. This looks different in the blend with $\mathbf{4}-\mathbf{C F}_{\mathbf{3}}$-Ph-DTTzTz in which the fast PL component ascribed to MDMO-PPV is not quenched while the slower one from the DTTzTz molecules was clearly reduced, corresponding rather to only hole transfer from the latter to the donor polymer. The negligible CT observed in MDMO-PPV:Th-DTTzTz blends then results from the very small driving force for charge transfer between LUMO levels as well as between HOMO levels of the two compounds. Indeed, values in the range of $0.3-0.5 \mathrm{eV}$ are considered necessary for efficient exciton splitting and charge separation in blends for 3D BHJ solar cells. ${ }^{5,6}$

Band-edge states have been revealed by optical absorption and EQE measurements as low-energy shoulders in the spectra, which in any case are related to the interaction between polymers and small molecules in the blends. One may assume that heteromolecular interactions lead to an increased order 
along the polymer chain resulting in an improved conjugation length and therefore a decreased HOMO-LUMO gap in the polymer. In such a case, the photocreated excitons can be expected to be rapidly trapped in regions with lower exciton energy and the PL to be shifted to lower energy, as is observed here. As an alternative, one could identify the shoulder with indirect excitonic states, the so-called CTC-complexes, in which hole and electron are localized on the donor and acceptor, respectively, at each side of the interface. In the last several years the importance of the CTC has been demonstrated for different BHJ donor-acceptor combinations. ${ }^{27,28} \mathrm{In}$ the blends studied here, the lowest bandgap material is the donor polymer and moreover one expects only small differences in LUMO energy between the donor and acceptor. Therefore, a CTC transition would be expected just below the MDMO-PPV bandgap, where we observe the absorption shoulder which was shown to contribute to the photocurrent. The shift to lower energy matches quite well with the decrease in LUMO level inferred from characterization in solution, correlating well with CT efficiency. The relatively large oscillator strength of this shoulder is however unexpected for such an indirect CTC-type transition. Further investigations are needed to distinguish between these two models.

The donor:acceptor combination selected for highest $\mathrm{CT}$ efficiency, MDMO-PPV:4-CN-Ph-DTTzTz, meets the expectations for a novel OPV material in several respects. First, we were able to demonstrate $\mathrm{CT}$ from either of the donor and acceptor component, thus offering the perspective of higher absorbance of the active layer. This could be a very favourable feature in a tandem solar cell for collection of the high-energy part of the solar radiation. Second, in solar cells this combination yields a $V_{\mathrm{oc}}=1.39 \mathrm{~V}$, much higher than in polymer: fullerene devices. The $V_{\text {oc }}$ is following the larger effective bandgap in this blend, with an energy loss of $\sim 0.6 \mathrm{eV}$ relative to the band edge, similar to what was found in other BHJ solar cells. ${ }^{5,6}$

Despite the demonstrated efficiency of CT after photon absorption in this blend, the corresponding solar cells have shown poor photovoltaic performance (maximum 0.1\% power conversion efficiency), directly resulting from low photocurrent generation. The temperature-dependent PIA measurements from $T=90 \mathrm{~K}$ to RT reveal an important difference between this material and the well-established MDMO-PPV:PCBM blend. In the latter much slower kinetics are observed at low temperature and moreover PIA persists up to RT, while in blends with 4-CN-Ph-DTTzTz as acceptor the response is becoming very fast above $T=200 \mathrm{~K}$ and PIA has vanished at RT, pointing to a much lower activation energy for recombination. This situation is similar to that in polymer-polymer blends reported by Morteani et $a l .{ }^{33}$ who exploited small energy level offsets between donor and acceptor compounds to obtain efficient OLED operation in a BHJ structure based on thermally activated transfer from charge separated states across the interface to exciton states in the polymers. Also, increased formation of the exciton in the lowest bandgap compound from initially separated carriers was recently reported on the basis of increased singlet exciton $\mathrm{PL}$ when the driving force for $\mathrm{CT}$ is decreasing in a series of solar cells with different donor-acceptor blends. ${ }^{29}$ The remaining
PL observed in our case at RT may be ascribed to a thermally activated back reaction to exciton states in one of the blend components, even in the best polymer:DTTzTz blend. In a broader perspective, it is not evident whether one will be able to reconcile low driving force with good solar cell efficiency, but good reason to pursue this approach may be found in a very recent report on which moderate internal quantum efficiencies could be demonstrated for a BHJ active layer with an energy offset of only $0.1 \mathrm{eV}^{30}$

Thermally activated carrier recombination will evidently be detrimental for the solar cell performance, but also other aspects should be taken into account. Based on previous data, the mobilities of the charge carriers in the pure materials, the MDMO-PPV as well as the DTTzTz compounds, are not expected to be the limiting factor for current generation. However, little is known yet about the morphology of the polymer:DTTzTz blends, which is an important factor to obtain efficient carrier transport to the respective electrodes. In relation to this, it has recently been reported for MDMOPPV:PCBM devices that PIA is detecting preferentially the localized polaron states in the polymer, while mobile polarons would rather be revealed by photoconductivity measurements. ${ }^{23}$ Therefore, the high PIA intensities we obtained may indicate trapping of the positive polarons, hampering hole transport and degrading the solar cell performance.

\section{Conclusions}

The combination of electron deficient substituted DTTzTz organic semiconductors with MDMO-PPV as acceptor and donor materials, respectively, has been shown to be a viable route to obtain blends with efficient CT. Three DTTzTz compounds were applied in this investigation, with varying acceptor character (as known from previous experimental and theoretical characterization). The CT efficiencies in the corresponding blends with MDMO-PPV were shown to be strongly correlated with increasing acceptor strengths.

A characteristic electronic transition was observed in each of the donor-acceptor blends at an energy just below the MDMO-PPV bandgap and it was shown that this transition contributes to the EQE in a MDMO-PPV : 4-CN-Ph-DTTzTz $(1: 1)$ solar cell device. The band-edge transition is induced by heteromolecular interactions between the polymer and the DTTzTz compounds, but further study is needed to distinguish between different models, in particular: (i) lowering of the polymer bandgap due to increased conjugation length, or (ii) indirect transitions between the polymer HOMO and DTTzTz LUMO.

The overall efficiency of BHJ solar cells based on these materials was found to be poor, even in the blend MDMOPPV:4-CN-Ph-DTTzTz selected for its highest CT efficiency. The temperature dependence of the response times measured in PIA points to thermal activation of charge recombination in these blends which would compete with the next steps in the photovoltaic process, i.e. the further separation and extraction of the carriers to the electrodes. Also, the high PIA signal measured at low temperature in this blend may indicate that the detected positive polarons in the polymer are in trapped states, which would lead to lower hole mobilities than expected in this reference donor material. 
Further work is now initiated to characterize the optical as well as electronic properties and the morphology of well-chosen blend materials combining various donor polymers with novel DTTzTz derivatives which have been designed and synthesized with the perspective of improved acceptor properties.

\section{Acknowledgements}

This work was realized with financial support from the IWT (Institute for the Promotion of Innovation by Science and Technology in Flanders) via the SBO-project 060843 'Polyspec', the Fund of Scientific Research-Flanders (FWO) (group project G.0555.10N) and the Hercules Foundation in its programme for support of instrumentation (contract AUHA013). We also thank BELSPO in the frame of the IAP P6/27 and IAP-7 FS2 networks.

\section{References}

1 T. W. Lee, N. S. Kang, J. W. Yu, M. H. Hoang, K. H. Kim, J.-I. Jin and D. H. Choi, J. Polym. Sci., Part A: Polym. Chem., 2010, 48, 5921.

2 S. Yoo, B. Domercq and B. Kippelen, Appl. Phys. Lett., 2004, 85, 5427; B. Ma, C. H. Woo, Y. Miyamoto and J. M. J. Fréchet, Chem. Mater., 2009, 21, 1413; H. H. P. Gommans, D. Cheyns, T. Aernouts, C. Girotto, J. Poortmans and P. Heremans, Adv. Funct. Mater., 2007, 17, 2653; Y. Terao, H. Sasabe and C. Adachi, Appl. Phys. Lett., 2007, 90, 103515; B. Walker, A. B. Tamayo, X.-D. Dang, P. Zalar, J. H. Seo, A. Garcia, M. Tantiwiwat and T.-Q. Nguyen, Adv. Funct. Mater., 2009, 19, 3063.

3 Y. Z. Lin, Y. F. Li and X. W. Zhan, Chem. Soc. Rev., 2012, 41, 4245.

4 R. Kroon, M. Lenes, J. C. Hummelen, P. W. M. Blom and B. De Boer, Polym. Rev., 2008, 48, 531; H. J. Son, F. He, B. Carsten and L. Yu, J. Mater. Chem., 2011, 21, 18934.

5 C. Deibel and V. Dyakonov, Rep. Prog. Phys., 2010, 73, 096401.

6 T. M. Clarke and J. R. Durrant, Chem. Rev., 2010, 110, 6736.

7 F. G. Brunetti, X. Gong, M. Tong, A. J. Heeger and F. Wudl, Org. Electron., 2010, 49, 532; J. E. Anthony, Chem. Mater., 2011, 23, 583; P. Sonar, J. P. Fong Lim and K. L. Chan, Energy Environ. Sci., 2011, 4, 1558; T. L. Zhou, T. Jia, B. N. Kang, F. H. Li, M. Fahlman and Y. Wang, Adv. Energy Mater., 2011, 1, 431; E. Ahmed, G. Q. Ren, F. S. Kim, E. C. Hollenbeck and S. A. Jenekhe, Chem. Mater., 2011, 23, 4563; J. T. Bloking, X. Han, A. T. Higgs, J. P. Kastrop, L. Pandey, J. E. Norton, C. Risko, C. E. Chen, J.-L. Brédas, M. D. McGee and A. Sellinger, Chem. Mater., 2011, 23, 5484; Y. Shu, Y. F. Lim, Z. Li, B. Purushothaman, R. Hallani, J. E. Kim, S. R. Parkin, G. G. Malliaras and J. E. Anthony, Chem. Sci., 2011, 2, 363; Y. Zhou, L. Ding, K. Shi, Y. Z. Dai, N. Ai, J. Wang and J. Pei, Adv. Mater., 2012, 24, 957; Y. Z. Lin, P. Cheng, Y. F. Li and X. W. Zhan, Chem. Commun., 2012, 48, 4773; K. Gui, K. Mutkins, P. E. Schwenn, K. B. Krueger, A. Pivrikas, P. Wolfer, N. T. Stutzmann, P. L. Burn and P. Meredith, J. Mater. Chem., 2012, 22, 1800 .

8 S. Ando, J.-i. Nishida, Y. Inoue, S. Tokito and Y. Yamashita, J. Mater. Chem., 2004, 14, 1787; Y. Fujisaki, M. Mamada, D. Kumaki, S. Tokito and Y. Yamashita, Jpn. J. Appl. Phys., 2009, 48, 111504; A. P. Naraso and F. Wudl, Macromolecules, 2008, 41, 3169; I. Osaka, G. Sauvé, R. Zhang, T. Kowalewski and R. D. McCullough, Adv. Mater., 2007, 19, 4160; I. Osaka, R. Zhang, J. Liu, D.-M. Smilgies, T. Kowalewski and R. D. McCullough, Chem. Mater., 2010, 22, 4191.

9 S. Van Mierloo, S. Chambon, A. E. Boyukbayram, P. Adriaensens, L. Lutsen, T. J. Cleij and D. Vanderzande, Magn. Reson. Chem., 2010, 48, 362.

10 S. Van Mierloo, K. Vasseur, N. Van den Brande, A. E. Boyukbayram, B. Ruttens, S. D. Rodriguez, E. Botek, V. Liégeois, J. D'Haen, P. J. Adriaensens, P. Heremans,
B. Champagne, G. Van Assche, L. Lutsen, D. J. Vanderzande and W. Maes, ChemPlusChem, 2012, 77, 923.

11 S. Van Mierloo, V. Liégeois, J. Kudrjasova, E. Botek, L. Lutsen, B. Champagne, D. Vanderzande, P. Adriaensens and W. Maes, Magn. Reson. Chem., 2012, 50, 379.

12 I. H. Jung, J. Yu, E. Jeong, J. Kim, S. Kwon, H. Kong, K. Lee, H. Y. Woo and H.-K. Shim, Chem.-Eur. J., 2010, 16, 3743; T. W. Lee, N. S. Kang, J. W. Yu, M. H. Hoang, K. H. Kim, J.-L. Jin and D. H. Choi, J. Polym. Sci., Part A: Polym. Chem., 2010, 48, 5921; S. K. Lee, J. M. Cho, Y. Goo, W. S. Shin, J.-C. Lee, W.-H. Lee, I.-N. Kang, H.-K. Shim and S.-J. Moon, Chem. Commun., 2011, 1791; L. Huo, X. Guo, S. Zhang, Y. Li and J. Hou, Macromolecules, 2011, 44, 4035; S. Subramaniyan, H. Xin, F. Sunjoo Kim and S. A. Jenekhe, Macromolecules, 2011, 44, 6245; J. Peet, L. Wen, P. Byrne, S. Rodman, K. Forberich, Y. Shao, N. Drolet, R. Gaudiana, G. Dennler and D. Waller, Appl. Phys. Lett., 2011, 98, 043301; Z.-G. Zhang, J. Min, S. Zhang, J. Zhang, M. Zhang and Y. Li, Chem. Commun., 2011, 9474; Y.-M. Hwang, J. Ohshita, Y. Harima, T. Mizumo, Y. Ooyama, Y. Morihara, T. Izawa, T. Sugioka and A. Fujita, Polymer, 2011, 52, 3912; M. Helgesen, M. V. Madsen, B. Andreasen, T. Tromholt, J. W. Andreasen and F. C. Krebs, Polym. Chem., 2011, 2, 253; E. Jeong, G.-h. Kim, I. H. Jung, P. Jeong, J. Y. Kim and H. Y. Woo, Curr. Appl. Phys., 2012, $12,11$.

13 S. Van Mierloo, A. Hadipour, M.-J. Spijkman, N. Van den Brande, B. Ruttens, J. Kesters, J. D'Haen, G. Van Assche, D. M. de Leeuw, T. Aernouts, J. Manca, L. Lutsen, D. Vanderzande and W. Maes, Chem. Mater., 2012, 24, 587.

14 N. S. Sariciftci, L. Smilowitz, A. J. Heeger and F. Wudl, Science, 1992, 258, 1474; R. A. J. Janssen, J. C. Hummelen, K. Lee, K. Pakbaz, N. S. Sariciftci, J. Heeger and F. Wudl, J. Chem. Phys., 1995, 103, 788.

15 Y. Wang, D. Kurunthu, G. W. Scott and C. J. Bardeen, J. Phys. Chem. C, 2010, 114, 4153.

16 K. Colladet, S. Fourier, T. J. Cleij, L. Lutsen, J. Gelan, D. Vanderzande, L. Huong Nguyen, H. Neugebauer, S. Sariciftci, A. Aguirre, G. Janssen and E. Goovaerts, Macromolecules, 2006, 40, 65; A. Aguirre, G. Janssen, E. Goovaerts, K. Colladet, D. Vanderzande and L. Lutsen, Eur. Phys. J.: Appl. Phys., 2006, 36, 285.

17 S. Bertho, G. Janssen, T. J. Cleij, B. Conings, W. Moons, A. Gadisa, J. D'Haen, E. Goovaerts, L. Lutsen, J. Manca and D. Vanderzande, Sol. Energy Mater. Sol. Cells, 2008, 92, 753.

18 L. Smilowitz, N. S. Sariciftci, R. Wu, C. Gettinger, A. J. Heeger and F. Wudl, Phys. Rev. B: Condens. Matter Mater. Phys., 1993, 47, 13835 .

19 V. Dyakonov, G. Zoriniants, M. Scharber, C. J. Brabec, R. A. J. Janssen, J. C. Hummelen and N. S. Sariciftci, Phys. Rev. B: Condens. Matter Mater. Phys., 1999, 59, 8019.

20 J. De Ceuster, E. Goovaerts, A. Bouwen, J. C. Hummelen and V. Dyakonov, Phys. Rev. B: Condens. Matter Mater. Phys., 2001, 64, 195206.

21 V. I. Krinichnyi, H.-K. Roth, S. Sensfuss, M. Schrödner and M. Al-Ibrahim, Physica E, 2007, 36, 98; V. I. Krinichnyi, P. A. Troshin and N. N. Denisov, J. Chem. Phys., 2008, 128, 164715; V. I. Krinichnyi and E. I. Yudanova, J. Phys. Chem. C, 2012, 116, 9189.

22 K. H. Lee, R. A. J. Janssen, N. S. Sariciftci and A. J. Heeger, Phys. Rev. B: Condens. Matter Mater. Phys., 1994, 49, 5781.

23 T. Offermans, S. C. J. Meskers and R. A. J. Janssen, Org. Electron., 2006, 7, 213; T. Offermans, S. C. J. Meskers and R. A. J. Janssen, Org. Electron., 2007, 8, 325.

24 B. Kraabel, J. C. Hummelen, D. Vacar, D. Moses, N. S. Sariciftci, A. J. Heeger and F. Wudl, J. Chem. Phys., 1996, 49, 4267; I.-W. Hwang, D. Moses, Z. G. Zhu, D. Waller, R. Gaudiana, C. J. Brabec and A. J. Heeger, Adv. Mater., 2007, 19, 2307; I.-W. Hwang, D. Moses and A. J. Heeger, J. Phys. Chem. C, 2008, 112, 4350; H. Ohkita and S. Ito, Polymer, 2011, 52, 4397.

25 C. M. Hill, Y. Zhu and S. Pan, ACS Nano, 2011, 5, 942; W. J. E. Beek, M. M. Wienk, M. Kemerink, X. Yang and R. A. J. Janssen, J. Phys. Chem. B, 2005, 109, 9505.

26 S. A. Zapunidi, Y. V. Krylova and D. Y. Paraschuk, Phys. Rev. B. Condens. Matter Mater. Phys., 2009, 79, 205.

27 K. Vandewal, A. Gadisa, W. D. Oosterbaan, S. Bertho, F. Banishoeib, I. Van Severen, L. Lutsen, T. J. Cleij, 
D. Vanderzande and J. V. Manca, Adv. Funct. Mater., 2008, 18, 2064.

28 D. Veldman, S. C. J. Meskers and R. A. J. Janssen, Adv. Funct. Mater., 2009, 19, 1939; K. Vandewal, K. Tvingstedt, A. Gadisa, O. Inganäs and J. V. Manca, Nat. Mater., 2009, 8, 904.

29 M. A. Faist, T. Kirchartz, W. Gong, R. S. Ashraf, I. McCulloch, J. C. de Mello, N. J. Ekins-Daukes, D. D. C. Bradley and J. Nelson, J. Am. Chem. Soc., 2012, 134, 685.

30 K. Vandewal, Z. Ma, J. Bergqvist, Z. Tang, E. Wang, P. Henriksson, K. Tvingstedt, M. R. Andersson, F. Zhang and O. Inganäs, Adv. Funct. Mater., 2012, 22, 3480.
31 S. Kuroda, T. Noguchi and T. Ohnishi, Phys. Rev. Lett., 1994, 72, 286; S. Kuroda, K. Murata, T. Noguchi and T. Ohnishi, J. Phys. Soc. Jpn., 1995, 64, 1363.

32 A. Aguirre, P. Gast, S. Orlinskii, I. Akimoto, E. J. J. Groenen, H. El Mkami, E. Goovaerts and S. Van Doorslaer, Phys. Chem. Chem. Phys., 2008, 10, 7129.

33 A. C. Morteani, A. S. Dhoot, J. Kim, C. Silva, N. C. Greenham, C. Murphy, E. Moons, S. Ciná, J. H. Burroughes and R. H. Friend, Adv. Mater., 2003, 15, 1708; A. C. Morteani, P. Sreearunothai, L. M. Herz, R. H. Friend and C. Silva, Phys. Rev. Lett., 2004, 92, 247402. 